\title{
European roadmap to the realization of fusion energy: mission for solution on heat-exhaust systems
}

\author{
M.Turnyanskiy ${ }^{\mathrm{a}}$, R. Neu ${ }^{\mathrm{b}, \mathrm{c}}$, R. Albanese ${ }^{\mathrm{d}}$, R. Ambrosino ${ }^{\mathrm{d}}$, C. Bachmann ${ }^{\mathrm{a}}$, S. Brezinsek ${ }^{\mathrm{e}}, \mathrm{T}$. Donne , $^{\mathrm{a}}$ \\ T. Eich ${ }^{b}$, G. Falchetto ${ }^{f}$, G. Federici ${ }^{a}$, D. Kalupin ${ }^{a}$, X. Litaudon ${ }^{a}$, ML. Mayoral ${ }^{a}$, DC. McDonald ${ }^{a}$, \\ H. Reimerdes ${ }^{\mathrm{g}}$, F. Romanelli ${ }^{\mathrm{a}}$, R. Wenninger ${ }^{\mathrm{a}}, \mathrm{J}^{-H}$. You ${ }^{\mathrm{b}}$ \\ ${ }^{a}$ EUROfusion PMU, Garching, Boltzmannstraße 2, D-85748, Garching, Germany \\ ${ }^{b}$ Max-Planck-Institut für Plasmapysik, Boltzmannstraße 2, D-85748, Garching, Germany \\ ${ }^{c}$ Technische Universität München, Fachgebiet Plasma-Wand-Wechselwirkung, D-85748, Garching, Germany \\ ${ }^{d}$ Assoc. EURATOM/ENEA/CREATE/DIETI - Univ. Napoli Federico II, Via Claudio 21, I-80125 \\ ${ }^{e}$ Association EURATOM/Forschungszentrum Jülich GmbH, 52425 Jülich, Germany \\ ${ }^{f} C E A$, IRFM, F-13108 Saint-Paul-lez-Durance, France \\ ${ }^{g}$ EPFL, CRPP, CH-1015 Lausanne, Switzerland
}

\begin{abstract}
Horizon 2020 is the largest EU Research and Innovation programme to date. The European fusion research programme for Horizon 2020 is outlined in the "Roadmap to the realisation of fusion energy" and published in 2012 [1]. As part of it, the European Fusion Consortium (EUROfusion) has been established and will be responsible for implementing this roadmap through its members. The European fusion roadmap sets out a strategy for a collaboration to achieve the goal of generating fusion electricity by 2050. It is based on a goal-oriented approach with eight different missions including the development of heat-exhaust systems which must be capable of withstanding the large heat and particle fluxes of a Fusion Power Plant (FPP). A summary of the main aims of the Mission for a solution on heat-exhaust systems and the EUROfusion consortium strategy to set up an efficient Work Breakdown Structure and the collaborative efforts to address these challenges will be presented.
\end{abstract}

Keywords: divertor, DEMO, EUROfusion, Roadmap, heat-exhaust

\section{Introduction}

The main challenge in realising a fusion power plant (FPP) is the adequate control of heat exhaust which is the main aim of Mission 2 of the European Fusion Roadmap [1]. Already demanding for ITER, the problem is amplified for a FPP where the assumed linear dimensions are $\approx 50 \%$ larger and the fusion power output at least 3 times higher (see for example [2]). The power crossing the magnetic separatrix is channelled along the magnetic field lines to the divertor where it is exhausted on actively cooled divertor targets. The heat flows in a narrow radial layer (SOL) of width $\lambda_{\mathrm{q}}(\sim 1 \mathrm{~mm}$ at the midplane for ITER) which scales only weakly with machine size [3]. This means that the loaded divertor area scales approximately with the major radius $\mathrm{R}$ of a device making $\mathrm{P} / \mathrm{R}$ ( $\mathrm{P}$ being the exhaust power) a crucial parameter when extrapolating to larger devices. On the current ITER exhaust assumptions $\approx 40 \%$ of the $\approx 150 \mathrm{MW}$ ITER heating power (fusion alpha particle power and auxiliary heating) is radiated inside the magnetic separatrix and $60 \%(\approx 90 \mathrm{MW})$ will flow into the SOL with $\approx 60 \mathrm{MW}$ (i.e. 2/3) towards the divertor outer target. In order to minimise the heat load, the divertor targets are inclined at a shallow angle to the magnetic field lines and are located in a region near the separatrix X-point with significant magnetic flux expansion, increasing the divertor target area in ITER to $\approx 2 \mathrm{~m}^{2}$. In the attached divertor regime, when almost all the heat entering the SOL ultimately ends up on the divertor target, this area increase lowers the power load to $\approx 30 \mathrm{MW} / \mathrm{m}^{2}$. However, despite significant progress during the last two decades, such heat loads exceed present technological capabilities - prototypes of watercooled copper alloys with either carbon or tungsten armour tested under cyclic power loads, have only demonstrated $\leq 20 \mathrm{MW} / \mathrm{m}^{2}$. These values are close to the intrinsic limits of the thermo-mechanical properties of the small number of materials suited for application in the fusion environment. In realistic fusion plant conditions these properties will be significantly degraded by neutron irradiation at the level of a few displacements per atom (dpas). Transients, tile misalignments and considerations of other realistic design tolerances will further reduce the power handling limits for reliable divertor target to $\approx 10 \mathrm{MW} / \mathrm{m}^{2}$, in the case of watercooled, and to even lower values in the case of $\mathrm{He}$ cooled components.

Solutions for the heat exhaust in DEMO/FPP are presently being explored along three main lines:

- Baseline divertor solution - a combination of radiative cooling and detachment. In such conditions a significant temperature gradient can be established and volume recombination of the plasma can take place, hence reducing the ion fluxes to the target;

- Innovative magnetic divertor configurations to achieve higher flux expansion, spreading the heat over a larger area or to achieve longer divertor connection lengths and larger divertor radiated power;

- Advanced plasma-facing components (PFCs) (e.g. liquid metals) that could exhaust higher heat loads. 
Low SOL temperature, associated with detached divertor conditions, also reduces the erosion of the divertor armour, the main factor defining its working life. The baseline divertor approach will be tested by ITER, thus providing an assessment of its adequacy for DEMO where limits are likely to be even more demanding on both SOL temperature and radial extend of detachment mainly due to much stricter erosion requirements.

Nevertheless, the risk of non-applicability of this solution for the high-confinement operation in the DEMO fusion reactor remains significant, potentially delaying the realization of fusion energy and prompting the search for alternative, risk mitigation solutions. Besides intensified research on divertor detachment in existing devices, the European fusion community discusses the plans for a Divertor Test Tokamak (DTT) which potentially could yield answers to the above mentioned questions in parallel to ITER operation. The strategy also includes a technological study of the feasibility and performance of water-cooled divertor targets concepts. It extends the ITER design and technology to DEMO relevant conditions (e.g., higher coolant temperatures and pressures and higher n-dose) and must be applicable to any potential divertor solid target concept. Finally, divertor pumping must be sufficient to exhaust the neutralised gas, most notably $\mathrm{He}$ ash, as well as limit the eroded impurities entering the main plasma. Any of the divertor acceptable solutions must satisfy these requirements together with those of heat exhaust.

\section{Baseline strategy}

\subsection{Detachment and radiating}

Detached divertor conditions have been obtained in several tokamaks and will be pursued by studies based on existing, especially all metal PFC, divertor devices (see for example [4]). The plasma detachment is normally characterized by a strong pressure gradient along magnetic field lines in front of the target and a reduced ion flux to the target in such a way that the plasma temperature close to the target decreases below several $\mathrm{eV}$ allowing volume recombination of the hydrogen isotopes. The required high collisionality can be achieved by: reducing the power flowing to the SOL $\left(\mathrm{P}_{\mathrm{SOL}}\right)$; increasing the SOL density; and producing magnetic configurations with a large connection length between the midplane and the divertor target. Decreased $\mathrm{P}_{\text {SOL }}$ can be achieved by radiating a large amount of power from the plasma edge (using extrinsic impurities). However, H-mode operation requires a minimum power to be conducted through the pedestal $\left(\mathrm{P}_{\text {thr }}\right)$ which will limit main chamber radiation specifically for ITER. In addition, the tungsten sputtering limit, which is largely determined by the impurity concentration in the divertor plasma, must also be evaluated [5]. Furthermore, detached conditions will have to be carefully controlled to ensure safe operation, requiring robust sensors, algorithms and actuators. ITER will play the ultimate role in proving the applicability of the "conventional" power exhaust scenario for DEMO [2] (Pulsed DEMO1: $\left.\mathrm{P}_{\text {fus }} \sim 1.8-2 \mathrm{GW}, \mathrm{f}_{\text {rad,core }} \sim 65 \%\right)$ but it can provide this information only after the successful achievement of long pulse high fusion gain $(\mathrm{Q} \approx 10)$ operation around 2030. In preparation of a safe ITER start-up and to provide further input for a decision on a DTT, the behaviour of detachment at high levels of heating power and radiation must be investigated during the first half of Horizon 2020. Specifically, the control of detachment, its compatibility with ELM mitigation and the behaviour close to the H-L threshold must be documented. Although divertor detachment has been achieved on present day tokamaks, its behaviour cannot be described by the existing numerical codes in a predictive fashion and must be supported by a strong model validation and code development efforts.

\subsection{Challenges of erosion of the PFC}

The PFC in the main chamber wall will receive power from radiation and particles and undergo erosion. For ITER, Be melting and excessive erosion can hamper operation whereas for DEMO the choice of the PFC material and the cooling technology depends critically on the particle spectrum and the total absorbed power. Therefore all solutions envisaged for the power exhaust in the divertor must also treat the main chamber issues in a consistent way. In addition to the power handling requirements of steady state and transient power loads (although not as demanding as in divertor targets), the erosion of the PFC has to be minimized in order to maximise the availability of the device and to reduce the deleterious effects of tritium co-deposition and dust production. To optimize the material choice specifically in the main chamber, the temperature and flux of plasma filaments must be quantified (including impurities). In parallel, improved PFC materials consistent with the engineering requirements must be developed. The specific plasma wall interaction (PWI) of seeding impurities with the respective armour material as well as the effect of material mixing will have to be determined. Since all conventional solutions foresee metallic PFCs, the effect of accidental melting by the plasma and on the performance of the component must be clarified

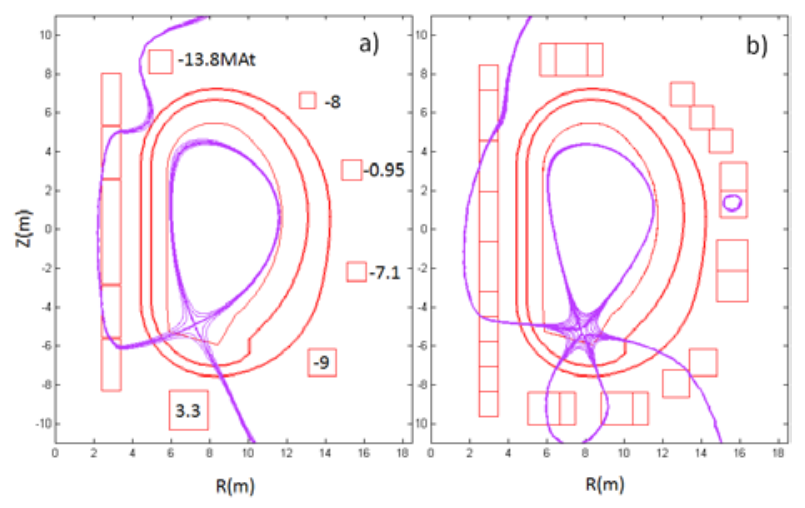

Fig. 1. Equilibrium reconstructions for DEMO with aspect ratio 3.1 using the CREATE code [6], [7]: a) conventional single null divertor; b) snowflake. The snowflake configuration has been obtained using a redundant PF coil system composed by 26 coils to provide a snowflake configuration for SOL analyses $\left(-24 \mathrm{MAt}<\mathrm{I}_{\mathrm{PF}}<14 \mathrm{MAt}\right)$. The optimization of the PF coil system in terms of dimensions, positions and currents taking account of port access, current density and load limits for the advanced configurations is planned for 2015. 


\section{Risk mitigation strategy}

A significant risk remains that high-confinement regimes of operation are incompatible with the larger core radiation fraction required in DEMO. Therefore, an investment in assessment of the adequacy for DEMO and proof-of-principle tests of innovative geometries as well as the use of liquid metals (LM) is required.

\subsection{Innovative divertor geometries.}

Two solutions are currently under investigation as alternatives for the conventional divertor: the "snowflake" [8] (see Fig. 1) and the "super-X" [9] configurations. Their benefit and limitations from the plasma physical point of view will be investigated during Horizon 2020 at a proof of principle level in small and medium size tokamaks. In addition, the extrapolability of both solutions to a FPP need to be assessed. Critical aspects are the complexity of the magnetic configuration and the necessity to avoid in-vessel coils in DEMO/FPP.

\section{Super-X Divertor:}

In a super-X divertor the poloidal field coils are used to deflect the SOL plasma into a separate divertor chamber. Moving the strike-point to larger $\mathrm{R}$ increases the geometric size of the plasma wetted area, reducing the target heat flux density. In addition, a quasi-null in the divertor poloidal magnetic field is generated, which increases the parallel connection length, allowing further power loss along the field lines.

\section{Snowflake Divertor:}

A snowflake diverted configuration is characterized by a second order null, i.e. not only the poloidal magnetic field vanishes (ordinary X-point) but also its first derivatives and the separatrix divides the poloidal plane into six sectors (giving the name to this configuration). The second-order null modifies the magnetic topology near the plasma boundary by expanding the flux 2-3 times more than in the ordinary X-point configuration. At the same time the connection length in that region increases, potentially reducing the local heat load to the divertor plates in a similar way as described above.

\subsection{Liquid Metal (LM)}

The use of LM as for both divertor and plasma facing materials inherently bears the advantage that they can simultaneously act as plasma facing surface and as cooling medium. LM-based solutions ( $L i, G a, S n)$ can potentially provide a very high heat load capability of up to several tens of $\mathrm{MW} / \mathrm{m}^{2}$ [10]. In addition, there is no neutron induced degradation and they offer relief on the problems of dust and erosion damage compared to solid surface solutions. In order not to dilute/contaminate the fusion plasma to a non-acceptable level, the erosion by sputtering and sublimation must be sufficiently low. This introduces demanding requirements for the operational temperature range to keep the vapour pressure at acceptably low level. Moreover, MHD forces, induced by currents in the moving liquid itself or by plasma transients, can distort the liquid surface in such a way that it no longer can provide its protective function. Depending on the technical solution, the cooling can be provided by the flowing liquid and/or by secondary conventional cooling. The assessment of LM based divertor solutions focuses on a conduction based capillary porous system (CPS), which is viewed as the least complex LM based solution. The difficulty of handling LM loops in a tokamak's vacuum environment means that any such system concept should be established as feasible with very high priority.

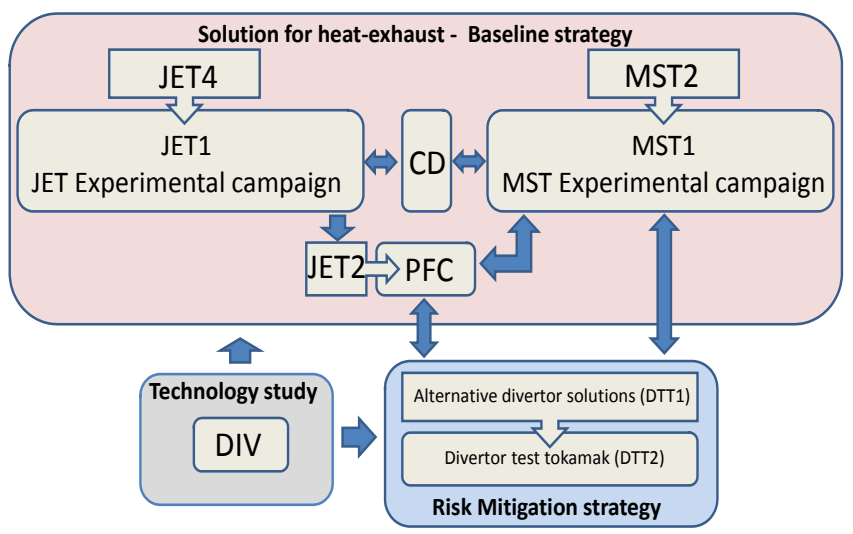

Fig. 2. Interconnection of Work Packages related to the

EUROfusion Mission 2: solution on heat-exhaust systems.

\section{Implementation}

A set of Work Packages (WPs) has been established by the EUROfusion consortium to solve the challenges of heat-exhaust for the DEMO fusion reactor addressed through the following programmatic headlines.

\section{1: Detachment control for ITER\&DEMO baseline strategy}

\section{2: Prepare efficient PFC operation for ITER\& DEMO} 2.3: Optimise predictive models for ITER\&DEMO
divertor/SOL

\section{4: Investigate alternative power exhaust solutions for DEMO}

The WPs can be divided into two main areas, as shown in Fig 2. The first area aims to demonstrate suitable schemes for controlled power exhaust in ITER and DEMO along the baseline divertor strategy and centres on experimental campaigns on both JET (WPJET1) and Medium-Size Tokamaks (WPMST1) providing direct ITER support. Both activities are supported by corresponding enhancement WPs (WPJET4, WPMST2) necessary for a successful exploitation in line with the headlines defined in the Road Map. Some more basic questions on the compatibility of $\mathrm{H}$-mode operation with high radiative power exhaust or investigations on alternative modes of operation (e.g. no/small ELM regimes) can be performed in a broader suite of tokamaks. A special set of WPs, namely WPCD: Code Development for Integrated Modelling and WPISA: Infrastructure Support Activities, will support the experimental campaigns by strong efforts on model validation concentrating on predictive tools for divertor/SOL and PWI modelling. For example, in this area, WPCD is focusing on developing: a faster SOLPS (2D fluid SOL, 3D neutral) code to accelerate validation, 3D SOL turbulence models and efficient integrated coreSOL models. A specific Work Package (WPPFC) will 
address the challenges of erosion of the PFCs for ITER and DEMO in order to maximize the availability of the devices and to reduce the deleterious effects of plasma contamination, hydrogen co-deposition and dust production. The test of PFC should be executed whenever possible - on high heat flux facilities and linear devices that avoid the complication of tokamak operation. WPPFC will be further complemented by the post mortem analysis of tiles removed from JET (WPJET2) and other MSTs to quantify and validate models for the rate of material erosion, transport and deposition.

Operation close to detachment and close to the H-L threshold as foreseen in ITER still requires considerable efforts investigating the confinement behaviour and control issues. Ultimately, ITER should demonstrate by 2030 the applicability of its baseline strategy to the power loads relevant for a reactor. In case of success and with further development of divertor modelling towards predictive capability, the baseline strategy can then be extrapolated and finally demonstrated in a DEMO FPP.

The baseline strategy can in principle only be fully evaluated together with achievement of the ITER $Q \approx 10$ milestone. Thus, in the absence of alternative solutions tested by 2030 at sufficiently large size and/or implementable in ITER, a failure of the baseline strategy would lead to a delay in the realization of fusion of $\approx 20$ years. Therefore, the second main area of investigation is devoted to the Mission 2 risk mitigating strategy. The investigation of alternative power exhaust solutions for DEMO exploiting innovative divertor configurations and solutions with liquid PFCs will be addressed in WPDTT1. For this reason, proof-of-principle European experiments are under construction or in operation in order to assess the capabilities of alternative divertor geometries (super-X: MAST-U, snowflake: TCV) and liquid metals. Given the early stage of development, it is essential that these concepts will need not only to pass the proof-of-principle test but they have to be closely accompanied by adequate modelling to assess their potential for extrapolation. Also, before being explored any further, the investigations must include the assessment of their technical and integration feasibility on DEMO perhaps by adjusting the overall DEMO system design to the concept. Since the extrapolation from proof-of-principle devices to ITER/DEMO based on divertor/edge modelling alone is considered too large, a dedicated test of the most promising concept must be performed in a larger device. It could be either implemented on an existing device (for example JET/JT-60SA (although their max P/R 10-12 is factor of two below that expected in ITER D-T phase) or on a dedicated Divertor Test Tokamak (DTT), entirely devoted to the divertor problem. DTT would be mainly aimed at the demonstration of innovative geometries and liquid metals at relevant $\mathrm{P} / \mathrm{R}$ and a scale that can be extrapolated directly to ITER and DEMO. In case that the baseline strategy turns out to be successful, this device could also contribute significantly to the investigations on detachment because of its envisaged capability to provide a large $\mathrm{P} / \mathrm{R}$.
A shortlist for the requirements of a possible DTT as a tool for risk mitigation for the DEMO exhaust has to be set-up early during Horizon 2020 in order to provide input for the decision on whether it should be built and for an eventual conceptual design phase which should start around the end of Horizon 2020. As part of the coherent Mission 2 approach, the definition of the exact scope and technical specifications of a DTT facility (either a new facility or the upgrade of an existing facility) will be addressed in a dedicated WP (DTT2: Definition and design of DTT facility) and, after a thorough review, a decision should be taken in 2016 for its construction. Preliminary design activities are already ongoing to understand whether these alternative solutions can be realistically integrated in a DEMO/FPP design, including the constraints arising from neutron shielding and remote maintenance.

In parallel to the physics investigations, the divertor targets technology solutions applicable for any chosen divertor concepts both conventional and advanced have to be qualified for FPP relevant conditions of higher coolant temperatures and pressures and higher n-dose. This work is addressed in WPDIV. An early DEMO probably will use copper-based water-cooled components (that will have to be tested on high heat flux test facilities) while in parallel progressing with the $\mathrm{R} \& \mathrm{D}$ on $\mathrm{W}$ alloys and $\mathrm{W}$ composites (WPMAT, see Mission 3 in [1]). If the $R \& D$ on new $W$ materials is successful, elements could possibly be implemented or tested on DEMO.

\section{Acknowledgments}

This work has been carried out within the framework of the EUROfusion Consortium and has received funding from the European Union's Horizon 2020 research and innovation programme under grant agreement number 633053. The views and opinions expressed herein do not necessarily reflect those of the European Commission.

\section{References}

[1] F. Romanelli et al., Fusion Electricity - A roadmap to the realisation of fusion energy, European Fusion Development Agreement (EFDA) 2012, ISBN 978-3-00040720-8

[2] G. Federici et al., Fus. Eng. Des., vol. 89, pp. 882-889, (2014).

[3] T. Eich et al., Nucl. Fusion 53 093031(2013)

[4] A. Kallenbach et al., Plasma Phys. Control. Fusion 55 124041 (2013)

[5] R. Wenninger, accepted by Nuclear Fusion, (2014).

[6] R. Albanese et al., Nuclear Fusion, Vol. 38 (1998), pp. 723-738.

[7] R. Albanese et al., Fus. Eng. Des., vol. 66-68, pp. 715718, (2003).

[8] F. Piras et al., Plasma Phys. Control. Fusion 51055009 (2009)

[9] A.W. Morris, IEEE Trans. Plasma Science 40682 (2012)

[10] J.W. Coenen et al, Physica Scripta T159 014037 (2014) 